sammenstellungen von Problemen und Lösungsmöglichkeiten beim Einsatz vernetzter PC's im Kontext rechtswissenschaftlicher Lehre vor allem aufi den folgenden Feldern:

\section{CIP.JUR: Lösung- sangebote}

- Lokale Netzwerke 'von PC's mit deren. Hardware-/SoftwareProblemen, insbesondere der Gewährleistung von Schutzrechten und der Datensicherung.

- Textverarbeitung als im juristischen Bereich besonders wichtiges Gebiet zum Einstieg in die Materie, auch mit gebietsspezifischen Überlegungen zur Funktionalität, Leistung und den Auswahlkriterien bei Textverarbeitungsprogrammen.

- Datenbankkonzepte und Standard-Datenbanksysteme vor dem Hintergrund juristischer Einsatzaufgaben.

- Informations- und Kommunikationssysteme und -dienste, - mit Diskussion spezieller Systeme im juristischen Bereich. - Methodische und kritische Würdigung von Entwicklungen im Gebiet von Autorensystemen und Expertensystem-Shells.

Zielsetzung war es dabei, jeweils in der Betrachtung von Beispielen generelle Probleme und Lösungsmöglichkeiten sichtbar $\mathrm{zu}$ machen.

Auf einer allgemeinen Ebene hat sich das Projekt besonders um zwei Aufgaben bemüht:

- einerseits um die Verknüpfungsmöglichkeiten handhabungsorientierter Einführungen in Hardware und Software mit den bisherigen, eher theoretisch orientierten Lehrinhalten der Rechtsinformatik,

- andererseits um die Einbrin-gung perspektivisch gesehener Anforderungen künftiger juristischer Praxis in den Kontext der

juristischen Ausbildung. Hier konnten vor allem langjährige Erfahrungen von Mitgliedern der Projektgruppe in Forschung und Entwicklung für den DVr Einsatz in der Justiz genutzt werden.

Die Ausführungen von Professor Fiedler waren geeignet, eine Vielzahl weiterer Überlegungen vor allem zur Rolle der Rechtsinformatik in den juristischen Fakultäten anzustoßen. Aus der Sicht des Berichterstatters verdienen dabei vor allem die folgenden Aspekte eine Hervorhebung.

Sicherlich ist es so, daß die Informatik als' generelles Grundwissen dringend gebraucht wird und deshalb in jeder Beziehung förderungswürdig ist. Es reicht aber bei weitem nicht aus, über das Computer-Investitions-Programm Mittel zur Beschaffung von Hard- und Software zur Verfügung zu stellen. Es bedarfi vielmehr einer Konkretisierung der Rolle, die die Informatik in Fächern wie der Rechtswissenschaft spielen soll. Dies gilt um so mehr, als die von Fiedler erwähnte Distanz der Rechtswissenschaft zur Informatik hier und da immer noch ausgeprägt ist. So liegt ein großes Kapital an Erfahrung und Wissen brach. Und dies, obwohl sich die Informatik als ,vierte Kulturtechnik" in allen Bereichen des gesellschaftlichen Lebens etabliert. Jedem Studenten sollte im Rahmen der Didaktik und Lehrziele des Fachbereiches ein PC zur Erhöhung seiner Kreativität und Produktivität zur Verfügung gestellt werden. Die Studenten, die heute nicht lernen mit dieser neuen Kulturtechnik umzugehen, laufen Gefahr, die Analphabeten von morgen sein. Es reicht daher nicht aus, einen noch so teuren PC-Saal im Rahmen des Computer-InvestitionsProgrammes zu installieren. Die Strukturen müssen dadurch verändert werden, daß man der Rechtsinformatik schöpferische Freiräume zubilligt. Nur speziell in der Rechtsinformatik ausgebildete Personen sollten über die anstehenden Projekte entscheiden. Es müssen didaktische Modelle erarbeitet werden, die die Informatik in die Rechtswissenschaft einführen. Begleitend muß eine Diskussion ermöglicht werden, die sich mit der Frage auseinandersetzt, was sinnvollerweise mit den Computern getan werden kann.

\section{Die Softwareentwick- lungen an der juristischen Fakultät Tübingen}

Gerhard Ringwald und Manfred Gerblinger referierten über den Tübinger Beitrag zur Rechtsinformatik

Seit 1971 werden an der juristischen Fakultät Tübingen Vorlesungen und Seminare in Rechtsinformatik, seit 1973 auch Programmierkurse angeboten. Es handelt sich um Grundlagenveranstaltungen im Sinne der JAPro. Seit 1984 besitzt die Fakultät eigene PCs, seit 1988 auch einen CIP-Pool.

Eigene Software-Lösungen werden seit Ende der siebziger Jahre entwickelt. Es handelt sich um Software von Juristen für Juristen, um Software, die der typischen juristischen Arbeit entgegen kommt. Zwei Programme wurden in besonderer Weise vorgestellt: DIALTUE(2) und TS.

\section{Der Tübinger Dialog (DIALTUE(2))}

DIALTUE(2) ist die Weiterentwicklung des bereits 1981 von Gerblinger (noch aufi einem Großrechner) programmierten Tübinger Dialogs. Diese Version wurde von Ringwald Mitte 1985 neu konzipiert und für PC's programmiert. Es handelt sich um ein Programm zur Ablage und zur Abfrage von Wissen. Vom Autor wird verlangt, sein Wissen in Textteile zu zerlegen und diese Textteile einzugeben. Das Programm verwaltet die Textteile automatisch in einer hierarchischen Baumstruktur. Textteile 


\section{Die Architektur} von DIALTUE

werden zu anderen Textteilen so in Beziehung gesetzt, als ob der Autor die Steuerung zwischen den Textteilen selbst geschrieben hätte. Das Programm unterscheidet zwischen Frage- und Informationstexten. Bei Fragetexten muß der Autor den Textteil für die mögliche Antwort JA und NEIN formulieren. Der Textteil für INFO (= weiß nicht) ist optional. Bei Informationstexten können bis $\mathrm{zu}$ viér andere Textteile (A, B, C, D) angebunden werden. An jeder beliebigen Stelle kann der Autor auf bereits formulierte Textteile springen und in der hierarchischen Baumstruktur Bindungen auch "quer" anlegen. Damit wird Wissen in ,vermaschten Bäumen" abgelegt. Es entstehen Dialogprogramme, die von anderen Nutzern durchlaufen werden können.

Die mit DIALTUE(2) erstellten Dialoge werden in Tübingen den Studenten als Lernprogramme überlassen. Einen weitaus größeren pädagogischen Erfolg bringt DIALTUE(2) in der Lehre indessen, wenn die Studenten mit diesem Programm selbst Dialoge erarbeiten. Denn dabei ist es nötig, das gewählte Rechtsgebiet sorgfältig $\mathrm{zu}$ strukturieren.

DIALTUE(2) ist ein Basisverfahren. Es könnte weiterentwickelt und um neue Funktionen ergänzt werden,

\section{DIALTUE: Ent- wicklungsperspekti-} ven etwa um die Anbindung an juristische Datenbanken. Die theoretischen Vorarbeiten sind bereits geleistet und laufen in Richtung eines Editors zur Erstellung elektronischer Kommentare.

\section{Der Tübinger Subsum- tionstrainer (TS)}

TS wurde Ende 1988 von Ringwald auf: Anregung von Fritjof: Haft entwickelt und programmiert (vgl. dazu die Vorstellung durch Ringwald, jur-pc-Newsletter, 10/89, S. 114 - 116).

Die Studenten können im PCPool vorgegebene Fälle auswählen. Sie beantworten dann vorgegebene Fragen zu diesen Fällen. Jeder Student kann sich alle $\mathrm{zu}$ einer Frage eingegebenen Antworten anzeigen lassen und sich für die seiner Meinung nach treffendste Antwort entscheiden. Die Antwort, für die am häufigsten votiert wurde, wird in eine Protokolldatei übernommen.

Das Programm wird in Tübingen zum juristischen „Subsumtionstraining" (derzeit im Straft recht) eingesetzt. Die Reihenfolge der Fragen zu einem juristischen Sachverhalt wird so gewählt, wie diese bei einer juristischen Fallösung auftreten. Dadurch trainiert der Student einerseits den Aufbau einer juristischen Fallösung, andererseits gewinnt er Einblick in die Gedankengänge seiner Kommilitonen.

Das Programm TS kann aber auch in der Weise eingesetzt werden, daß die Studenten zunächst gemeinsam die Gliederung des Falles erörtern und sich auf die Gliederungspunkte und die entsprechenden Fragen festlegen. Über das Masterprogramm werden die Fragen dann eingegeben. Bei dieser Einsatzweise sollte unbedingt eine Lehrkraft mit den Studenten die Fallgliederung besprechen.
Praxisnahe Ausbildung an EDV-Systemen der Justiz: Ein Bericht aus Bayern

Franz Göttlinger vom Bayerischen Staatsministerium der Justiz befaßte sich in seinem Vortrag mit dem Thema, wie die bayerische Justiz das Thema „EDV“ nen, wie etwa Rechtsreferendaren oder Beamtenanwärtern, nahe bringt.

\section{Stand der EDV in der bayerischen Justiz}

Die Ausstattung der Gerichte und Staatsanwaltschaften mit EDV, beruht in der bayerischen Justiz etwa seit dem Jahre 1984 überwiegend auf Systemen mit dem Betriebssystem UNIX (bzw. SINEX). Für die einzelnen Auft gabenbereiche der Gerichte und Staatsanwaltschaften muß jeweils spezielle Software entwickelt werden. Die Verfahren umfassen hauptsächlich den Bereich der Geschäftsstellen-und Kanzleitätigkeit, also die administrativen Hilfstätigkeiten für die Rechtspflegeorgane. Zum Teil sind aber diese selbst schon in das Verfahren integriert.

\section{Aus- und Fortbildung der Bediensteten in der bayerischen Justiz: Die nötige Infrastruktur.}

Die Einführung der Datenverarbeitung in größeren Umfang in einem Behördenbereich, der bisher damit praktisch nicht befaßt war, macht erhebliche Anstrengungen für die Aus- und Fortbildung des Personals notwendig. Die Entscheidung fiel dahin, die Aus- und Fortbildung im Justizbereich selbst zu organisieren. Ungünstig wirkte sich aus, daß Dienstanfänger in allen Lauft bahnen keinerlei Vorkenntnisse auf dem Gebiet der EDV، mitbringen. 
3. Ausbildung der Studierenden an der bayerischen Beamtenhochschule (Fachbereich Rechtspflege)

Die Beamtenanwärter für den gehobenen Justizdienst erhalten ihre fachtheoretische Ausbildung als Studierende an der Beamtenfachhochschule. Seit einigen Jahren werden für die Studieren EDV-Fachseminare (Dauer eine Woche) durchgeführt, in denen Grundlagen der Datenverarbeitung vermittelt und Anwendungsverfahren demonstriert werden. Für die Zukunft ist geplant, die allgemeine Einführung in die Informatik und die anwendungsbezogene Einführung in die Studiengänge bei der Beamtenfachhochschule zu integrieren.

\section{Ausbildung der übri- gen Beamtenanwärter an der bayerischen Justiz- schule}

Auch für die Beamten des mitt- leren Justizdienstes werden bisher einwöchige EDV-Seminare veranstaltet. Hier ist ebenfalls geplant, die allgemeine EDVEinführung und die praktische Unterweisung für die Anwendungsverfahren in den Unterricht $\mathrm{zu}$ integrieren.

\section{Einführung der Rechts- referendare in die EDV}

Der Umstand, daß an den Universitäten offensichdich die Einführung der Jura-Studenten in die EDV sehr unterschiedlich gehandhabt wird, machte ein Konzept für die Heranführung der Referendare an die EDV nötig. Wegen der in allen künftigen Berufsfeldern absehbaren Bedeutung der EDV erschien es - zweckmäßig, den Referendaren die nötigen Grundlagen im Rahmen der Arbeitsgemeinschaften zu vermitteln. Die bayerische Justiz entschied sich dafür, hier die gleiche Technik zugrundezulegen, die auch beim Einsatz in der bayerischen Justiz Anwendung fndet.

\section{Aus- und Fortbildungs- kurse für die Richter und alle Beamtenlaufbahnen in der bayerischen Justiz}

Neben der Einführung der Berufsanfänger ist es notwendig, die große Zahl der Richter, Beamten und Angestellten, die in ihrer Behörde mit der EDV in Berührung kommen, entsprechend auszubilden. Hierfür wurde ein System von Lehrgängen, kombiniert mit Schulung am Arbeitsplatz, entwickelt.

JULE - ein (nicht nur) juristisches Lern- und Datenbankprogramm

In einer eindrucksvollen Vorführung stellte Tony Moeller von der Universität des Saarlandes (Lehrstuhl für Rechtsinformatik, dort auch nähere Auskünfte) das von ihm entwickelte Lern- und Datenbankprogramm JULE vor (vgl. jur-pc-Newsletter $3 / 89$, s. $32 \mathrm{ft}$ und zum neuesten Stand jur-pc-Newsletter, $10 / 89, \quad S$. 116f).

Das JULE-System richtet sich vornehmlich an den Jura-Studenten, kann aber durch sehr einfache, vom Anwender selbst vorzunehmende, Umstrukturierungen so verändert werden, $\mathrm{da}$ auch Studenten anderer Fachbereiche, wie etwa Naturwissenschaftler, das Programm gewinnbringend einsetzen können. Programmierkenntnisse sind nicht erforderlich. Seinen innovativen Charakter macht das Programm beim ersten Hinsehen vor allem dadurch deutlich, daß man Teilfunktionen des Programmes über ein $\mathrm{Mi}$ krophon mit der Sprache steuern kann. Selbstverständlich ist auch eine reine Tastatur-Bedienung möglich. JULE läuft auf jedem IBM-kompatiblen Computer mit mindestens 512 KByte RAM und zwei 360 KByte-Diskettenlaufwerken. 1st Card - eine Expertensystem-Shell für den juristischen Anwender

1st Card ist eine Eigenentwicklung von Gerhard Oppenhorst (Forschungsstelle für juristische Informatik und Automation der Universität Bonn, Lennéstr. 35, 5300 Bonn 1).

Das Programm ist eine Expertensystem-Shell, die die „Programmierung “ von Expertensystemen ohne Programmierkenntnisse lediglich mit der "Maus" bereits nach kurzer Einarbeitungszeit ermöglicht. Es läuft aufi allen ATARI ST Computern im Monochrome-Betrieb unter GEM, sowohl von Diskette, als auch von Festplatte oder RAM-Disk. Das Programm wurde bereits in jur-pc besprochen $(5-6 / 1989$, S. 171 - 173).

\section{Der elektronische Kom-} mentar (ELEKOM)

Frau Brinkhoff von der Forschungsstelle für Rechtsinformatik der Philipps-Universität Marburg präsentierte ein Dokumentations- und Subsumtionssystem: ELEKOM.

ELEKOM faßt die Teilprogramme unter einer einheitlichen Benutzeroberfläche zusammen. Die Einzelprogramme, die von Studenten im Rahmen mehrerer Proseminare zur Rechtsinformatik erstellt wurden, dokumentieren verschiedene Tatbestände des besonderen Teils sowie ausgewählte Problembereiche aus dem allgemeinen Teil des Strafgesetzbuches. Gesteuert werden die Teilprogramme durch ein selbsterklärendes Menüsystem. Die Programme wurden in Basic und Pascal erstellt und in kompilierter Form in das Menüsystem eingebunden. Sie unterscheiden sich - je nach Ausbildungsstand der Autoren - in ihrer Komplexität und Benutzerfreundlichkeit. Die Mehrzahl der Programme stellt über eine menügesteuerte Benutzerführung der juristischen Subsumtionsmethode entsprechend - zunächst 